\title{
Usefulness of mortality data in determining the geography and time trends of dementia
}

\author{
C N MARTYN AND E C PIPPARD \\ From the MRC Environmental Epidemiology Unit (University of Southampton), Southampton General Hospital, \\ Southampton SO9 $4 X Y$
}

SUMMARY Fewer than $25 \%$ of people diagnosed during life as being demented were found to have this diagnosis coded as the underlying cause of death. In a sample of deaths certified as due to dementia the majority were found to have occurred in long-stay institutions. This distorts the geographical pattern of mortality because the Office of Population Censuses and Surveys (OPCS) considers these institutions to be the patient's usual address six months after his admission. Analysis of all certified deaths from dementia during 1968-78 by place of residence shows that areas with a significantly high SMR usually contain a large psychiatric hospital. Changes in diagnostic fashion and in the procedure by which OPCS selects the underlying cause of death have also affected numbers of deaths coded as dementia. Death certificate data are unlikely to be useful in examining either geographical variation or time trends in rates of dementia.

Detailed analysis of mortality data for England and Wales, by usual place of residence of the deceased, demonstrated considerable geographical variation in rates of death certified as due to dementia. ${ }^{1}$ Most of the deaths occurred in people over the age of 65 years. Evidence from necropsy series ${ }^{2}$ indicates that the commonest condition underlying dementia in this age group is Alzheimer's disease. This disease is now a serious cause of morbidity in the elderly population. Estimates of prevalence vary widely ${ }^{3}$ but, in a synthesis of all recent surveys, a rate of about $3 \%$ was proposed as the prevalence of moderate and severe dementia in people aged over 65 years in the UK. ${ }^{4}$ Population projections indicate that the numbers of elderly people will continue to rise until well into the next century, and the number of elderly demented is likely to become an increasing burden on health and social services.

We hoped that the geographical pattern of mortality might provide clues about environmental agents relevant to the causation of Alzheimer's disease. As a preliminary to interpreting this geographical pattern we investigated the usefulness of data derived from death certificates as an estimate of variations in mortality from dementia with geography and time.

\section{Methods}

The investigation was carried out in four separate parts.
BRIGHTON CLINIC STUDY

Using the diagnostic register of a psychogeriatric clinic (Brighton Clinic in Newcastle General Hospital) we identified 197 patients who had been diagnosed as having dementia in the years 1980 and 1981. All diagnoses had been made by a consultant psychogeriatrician. By 1986, when this study was carried out, 140 of these patients had died. The Office of Population Censuses and Surveys (OPCS) was able to trace and provide copies of the death certificates for 137 of the 140. These were analysed for underlying cause of death and other recorded conditions. The purpose of this study was to determine the proportion of demented patients who had a diagnosis of dementia recorded on the death certificate.

STUDY OF DEATHS CERTIFIED AS DUE TO DEMEN TIA

OPCS also provided 200 death certificates on which the underlying cause of death had been coded to the International Classification of Disease (ICD) ${ }^{5}$ numbers 290.0 and $290 \cdot 1$ (senile dementia and presenile dementia respectively) in people under the age of 75. These represented a 1 in 2 sample of all certificates with these codes for underlying cause of death, for 1978. Other recorded conditions contributing to death and place of death were analysed.

GEOGRAPHICAL VARIATION IN MORTALITY The mortality data for 1968-78 consisting of extracts from all death certificates for England and Wales were 
re-examined. Mortality rates for each sex for each local authority area were calculated from 1971 census data, which we grouped according to pre-1974 local authority boundaries. Death rates were expressed as standardised mortality ratios (SMRs).

\section{TIME TRENDS}

Published mortality statistics for dementia and related diagnoses $^{6}$ (ICD 290, 299, and 794, 8th revision; ICD $290,298.9,331$, and 797,9 th revision) were used to examine possible time trends.

\section{Results}

BRIGHTON CLINIC STUDY

Twenty of the 137 death certificates, all for deaths that had occurred between May 1981 and February 1982, had not been coded by OPCS. Of the remaining 117 patients, only $26(22 \%)$ had pre-senile or senile dementia (ICD 290) coded as the underlying cause of death. Dementia or a related diagnosis had been recorded under either part 1 or part 2 in 78 of the 137 $(57 \%)$ death certificates. When dementia had been recorded under part 1 the underlying cause of death was coded as pre-senile or senile dementia in most cases ( 15 of 18). When dementia appeared under part 2 it was very rarely ( 1 out of 20 ) coded as the underlying cause if the death had occurred before 1984. After 1984 senile or pre-senile dementia was coded as the underlying cause of death from part 2 more frequently (10 out of 17). Where the doctor certifying death had used the term dementia without qualifying it with 'senile' or 'pre-senile' the underlying cause of death became unspecified psychosis (ICD 299, 8th revision;

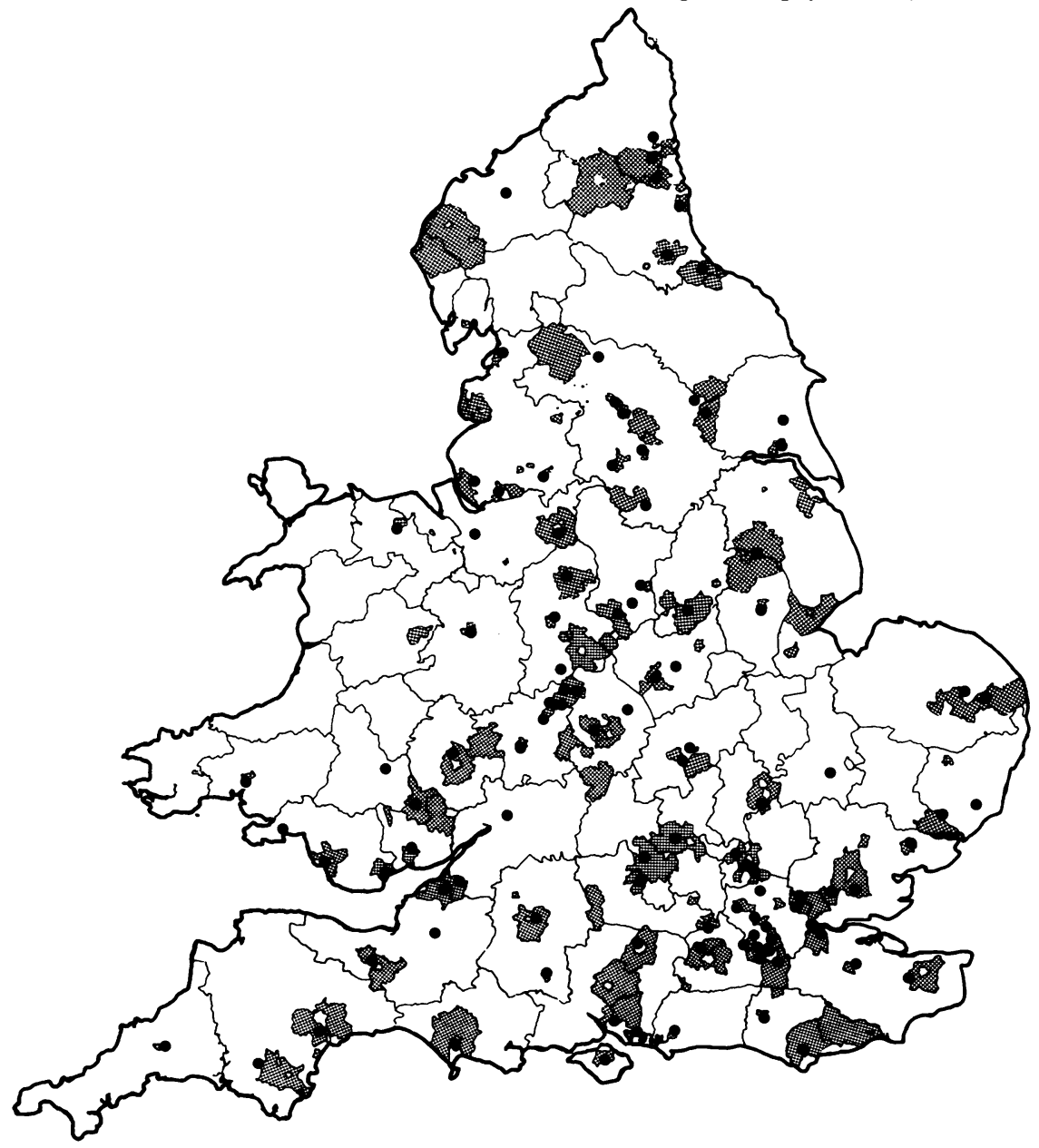

Fig 1. The geography of mortality from dementia. Local authority areas with significantly higher than average (p<0.05) $S M R S$ for men or women for senile dementia and with more than five deaths during the period 1968-78 are shown (dotted). The larger black dots show the locations of all psychiatric hospitals with more than 200 long-stay beds. 
298.9 , 9th revision) instead of pre-senile or senile dementia. Overall, men were less likely than women (6 out of 40 men against 20 out of 77 women) to have pre-senile or senile dementia coded as the underlying cause of death.

Place of death was an important determinant of whether a diagnosis of dementia was recorded on death certificates. Patients dying in psychiatric hospitals were more likely ( 30 out of 38 ) to have dementia recorded than those dying in general hospitals (29 out of 56) or at home or in part III accommodation (20 out of 43 ).

\section{STUDY OF DEATHS CERTIFIED AS DUE TO DEMENTIA}

Out of 200 death certificates in which the underlying cause of death was coded to senile or pre-senile dementia, the place of death had been a hospital in 168 cases $(84 \%)$. In 117 deaths $(59 \%)$ the terminal event, appearing under part la of the death certificate, was bronchopneumonia. Cardiac failure $(\mathbf{7 \%})$ and multiple pressure sores $(4 \%)$ were the other common conditions recorded under part la.
GEOGRAPHIC VARIATION IN MORTALITY

The map of England and Wales (fig 1) shows those local authority areas where the SMR for dementia was significantly above average $(p<0.05)$ and in which more than five deaths from dementia were recorded during 1968-78. Also shown on the map are the locations of all psychiatric hospitals with more than 200 long stay beds. A high degree of coincidence between the areas with high SMRs for dementia and the locations of psychiatric hospitals is apparent.

\section{TIME TRENDS}

The annual numbers of deaths from senile dementia and senility for 1974-84 are shown in figure 2. Between 1974 and 1983 a gradual rise in numbers of deaths from senile dementia mirrors a gradual fall in the number of deaths from senility. In 1984, a striking rise in the number of deaths from dementia occurred; increases of more than twofold were seen for both men and women.

Discussion

In a group of patients who were diagnosed at a $\frac{0}{0} \overbrace{}^{N}$

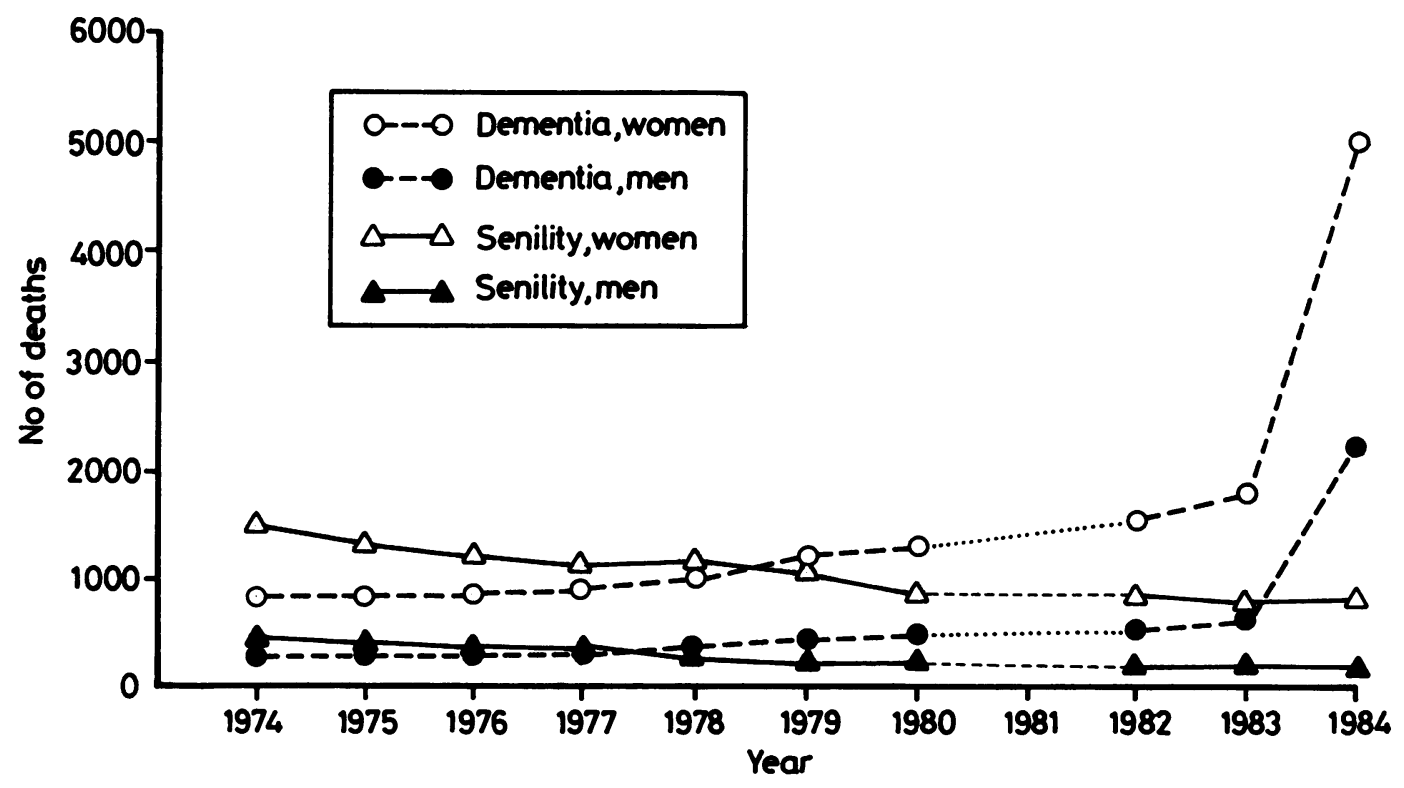

Fig 2. Annual numbers of deaths certified as senile dementia (ICD 290.0) and senility (ICD 794, 8th revision; 797 , 9 th revision ${ }^{8}$ ) in England and Wales 1974-84. No data for 1981 were available. 
specialised clinic as having dementia and who subsequently died, less than $25 \%$ had this diagnosis coded as the underlying cause of death. In only $58 \%$ of the patients did dementia appear anywhere on the death certificate. These observations were made in a city where psychiatrists and geriatricians have had an active research interest in dementia for many years. In other areas of England and Wales it is likely that dementia is recorded on death certificates with an even lower frequency.

We found, in the sample of death certificates from OPCS, that the majority of patients in whom the underlying cause of death was coded as senile or pre-senile dementia died in hospital. Hospitals with long stay beds are considered to be the patient's usual address by OPCS if the patient has been living there for more than six months before the date of death. ${ }^{6}$ Thus, those local authority areas which contain a hospital with a large number of long-stay beds are likely to show elevated SMRs for chronic neuropsychiatric disorders. The remarkable coincidence of the locations of large psychiatric hospitals with areas with high SMRs for dementia demonstrates the extent to which this practice has affected the geographical distribution of mortality from dementia.

In 1984, OPCS changed their procedure for implementing World Health Organisation Rule 3 regarding the selection of the underlying cause of death from the conditions listed in parts I and II of death certificates. ${ }^{6}$ This change affected death certificates where, under part la, the proximate cause of death was recorded as bronchopneumonia, and where a major disease was recorded in part II. Until 1984 the disease mentioned in part II would have been ignored for the purposes of coding the underlying cause of death. From 1984 onwards the disease mentioned in part II became the underlying cause of death. Because bronchopneumonia is a common terminal event in patients with dementia this change had the effect of more than doubling the mortality rate from dementia over a single year (fig 2). A more gradual increase in the number of deaths from dementia occurred steadily over the period 1974-83. This coincided with a slow decrease in the number of recorded deaths from senility. Its seems probable that these trends are partly explained by changes in diagnostic fashion. Some increase in numbers dying from dementia would also be expected from the changing age structure of the population.

We conclude that because of the low rate of recording of senile dementia as a cause of death and the distorted geographical pattern of the disease, death certificate data are not useful in exploring geographical variations in the frequency of dementia in England and Wales. Possible variations in diagnostic terminology over time and the change in the way WHO Rule 3 has been applied limit any inferences that can be drawn from changes in mortality rates from dementia with time. For all these reasons, predictions of the size of the demand on health services created by senile dementia will be misleading if they have been derived from death certificate data.

We thank Dr Gary Blessed, of the Brighton Clinic, Newcastle General Hospital, for allowing us to use his records, OPCS for providing us with death certificates, Dr Clive Osmond and other members of the MRC Unit for their helpful comments and Mrs Brigid Howells for typing the manuscript.

\section{References}

${ }^{1}$ Gardner MJ, Winter PD, Barker DJP. Atlas of mortality from selected diseases in England and Wales 1968-1978. Chichester: Wiley, 1984.

2 Tomlinson BE, Blessed G, Roth M. Observations on the brains of demented old people. J Neurol Sci 1970; 11: $205-42$.

${ }^{3}$ Henderson AS. The epidemiology of Alzheimer's disease. Br Med Bull 1986; 42: 3-10.

${ }^{4}$ Ineichen B. Measuring the rising tide. Br J Psychiatry 1987; 150: 193-200.

${ }^{5}$ World Health Organisation. International classification of diseases 1965. 8th Revision, London: HMSO, 1967.

${ }^{6}$ Office of Population Censuses and Surveys. Mortality statistics (cause), 1974-84, England and Wales. Series DH 5, No. 1-11. London: HMSO, 1975-85.

${ }^{7}$ World Health Organisation. International classification of diseases 1975. 9th revision. London: HMSO, 1977.

Accepted for publication December 1987 